\title{
Stage IIIA Hilar Cholangiocarcinoma AJCC v7
}

National Cancer Institute

\section{Source}

National Cancer Institute. Stage IIIA Hilar Cholangiocarcinoma A/CC v7. NCI Thesaurus. Code C88061.

Stage IIIA includes: T3, NO, MO. T3: Tumor invades unilateral branches of the portal vein or hepatic artery. N0: No regional lymph node metastasis. MO: No distant metastasis. (from AJCC 7th Ed.) 